\title{
Electronic Structures and Optical Absorption of N-type Conducting Polymers at Different Doping Levels
}

Sarbani Ghosh, Viktor Gueskine, Magnus Berggren, and Igor V. Zozoulenko*

Department of Science and Technology, Linköping University, SE-601 74 Norrköping, Sweden

E-mail: igor.zozoulenko@liu.se

Supplementary Information 

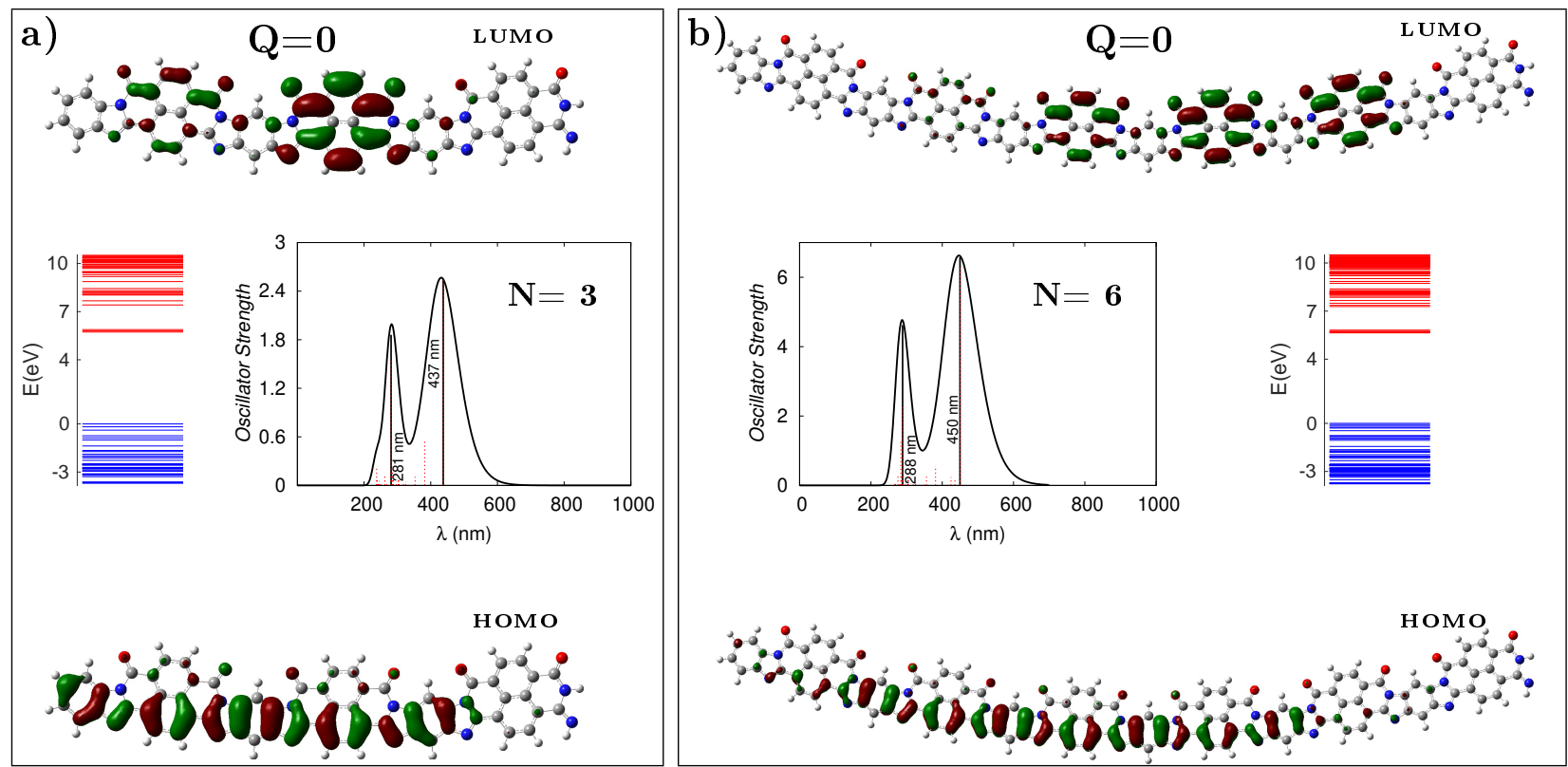

Figure S1: Band diagrams with HOMO/LUMO plots and the UV vis/NIR absoption spectra of undoped $(\mathrm{Q}=0)$ BBL chain with chain length of a) 3 monomer units $(\mathrm{N}=3)$ and b) 6 monomer units $(\mathrm{N}=6)$.
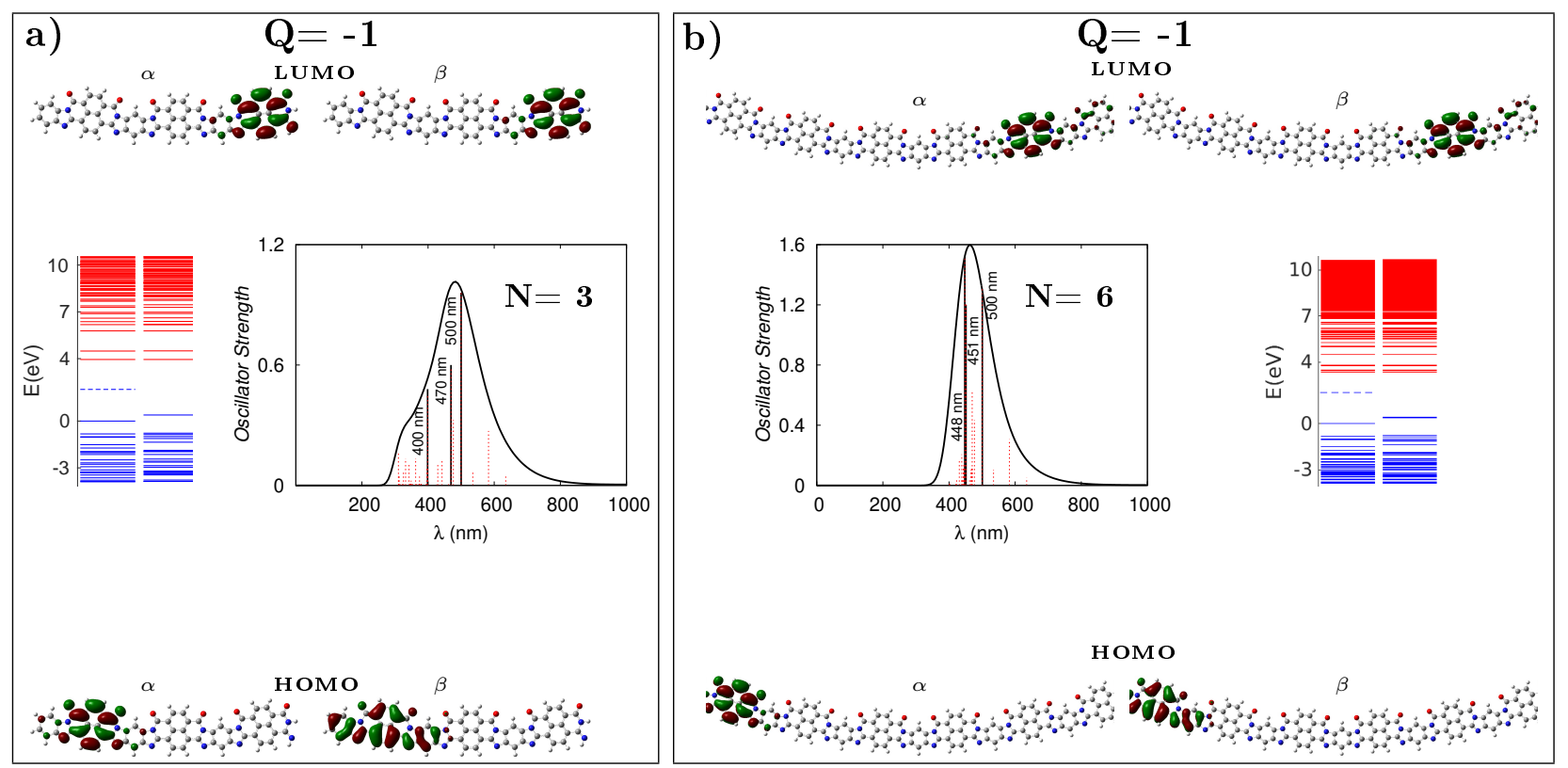

Figure S2: Band diagrams with HOMO/LUMO plots and the UV vis/NIR absoption spectra of doped $(\mathrm{Q}=-1)$ BBL chain with chain length of a) 3 monomer units $(\mathrm{N}=3)$ and b) 6 monomer units $(\mathrm{N}=6)$. 


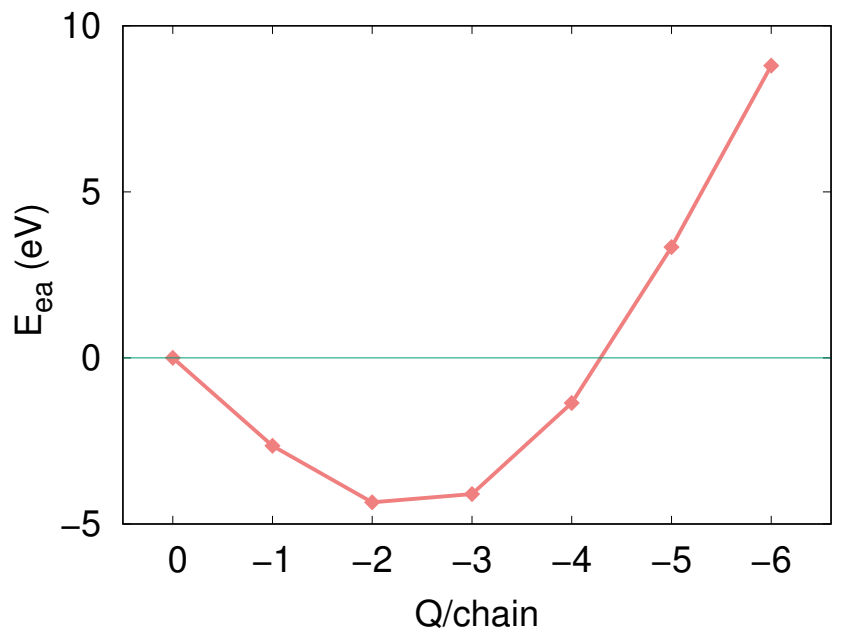

Figure S3: Electron affinity $\left(\mathrm{E}_{e a}\right)$, calculated by substracting the total energy of the particular system $(\mathrm{Q}=0$ to -6$)$ from the total energy of the neutral BBL chain, as a function of charge (Q) per chain is plotted. 
a)

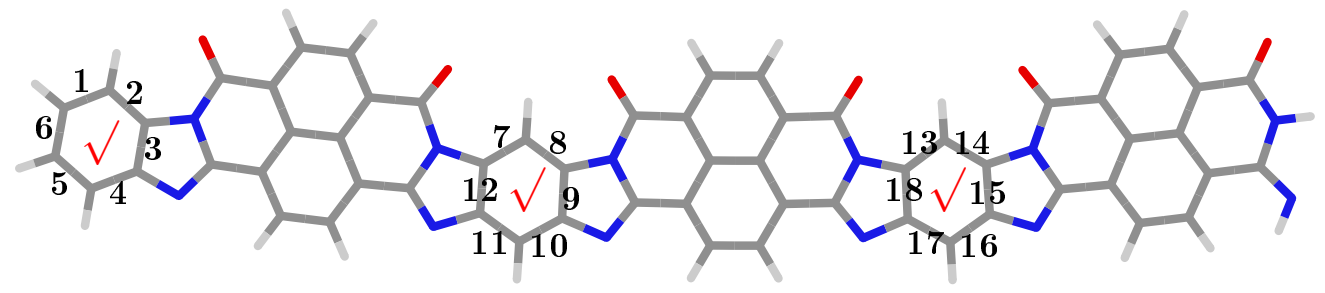

b)
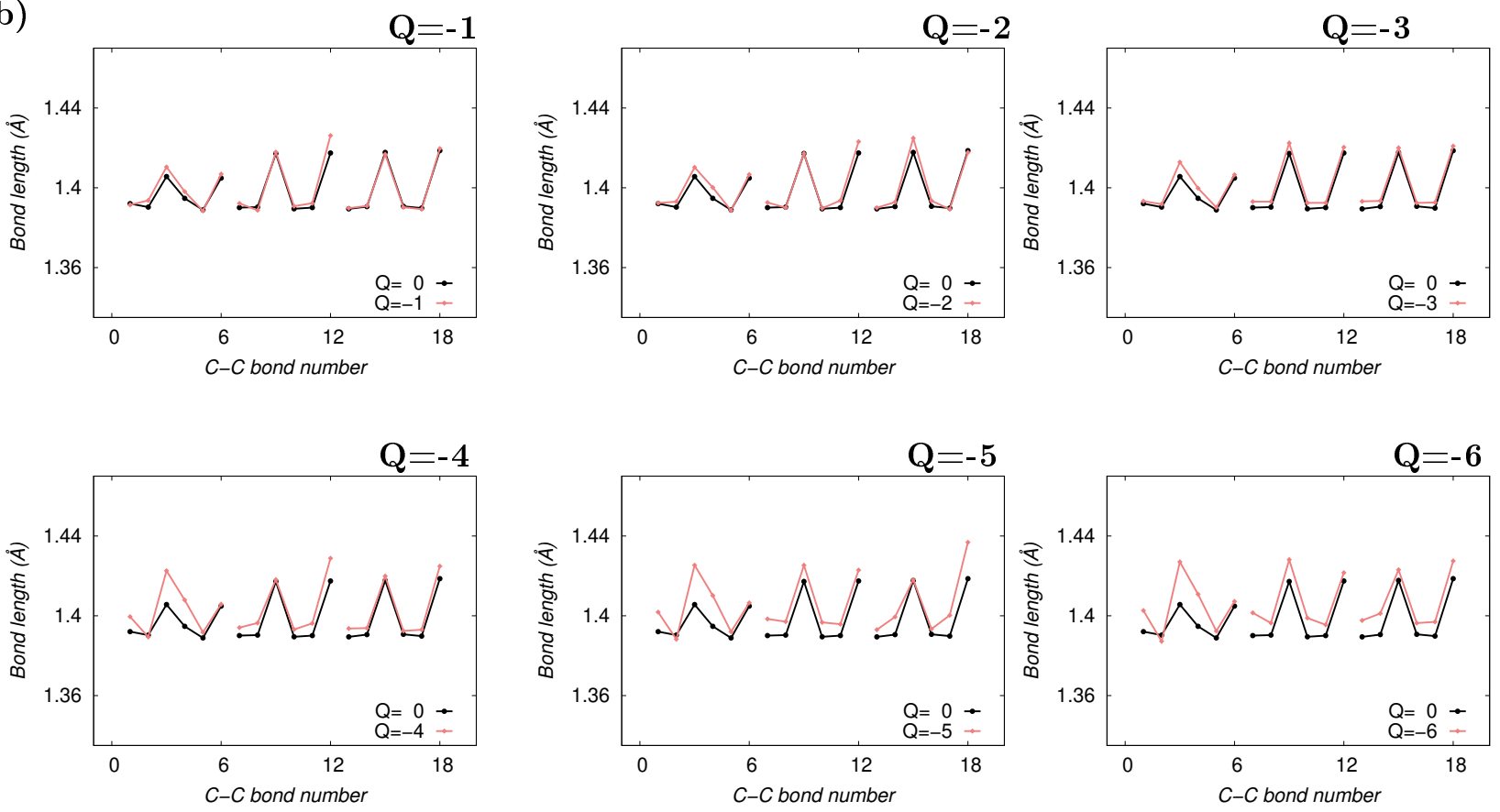

Figure S4: a) BBL chain where the benzene ring are marked by red tikz and the $\mathrm{C}$-C bond number is also shown, b) Bond length alternation of the benzene ring from $\mathrm{M}_{2}$ repeating unit of doped BBL $(\mathrm{Q}=-1$ to -6$)$. 
a)

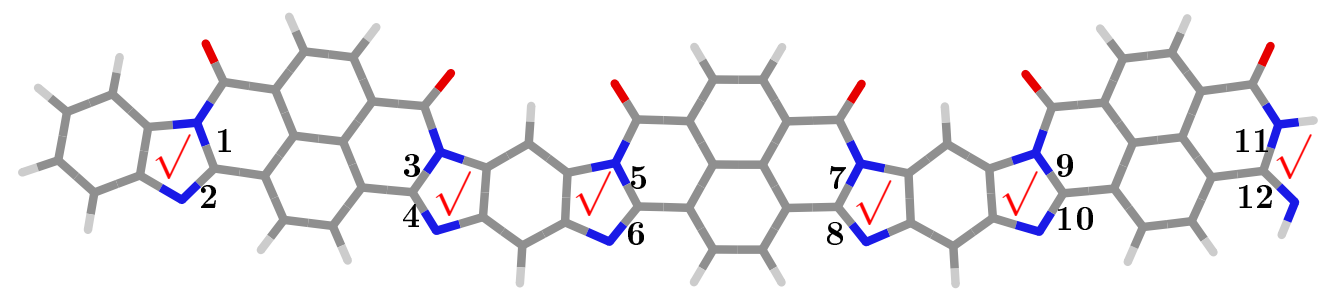

b)
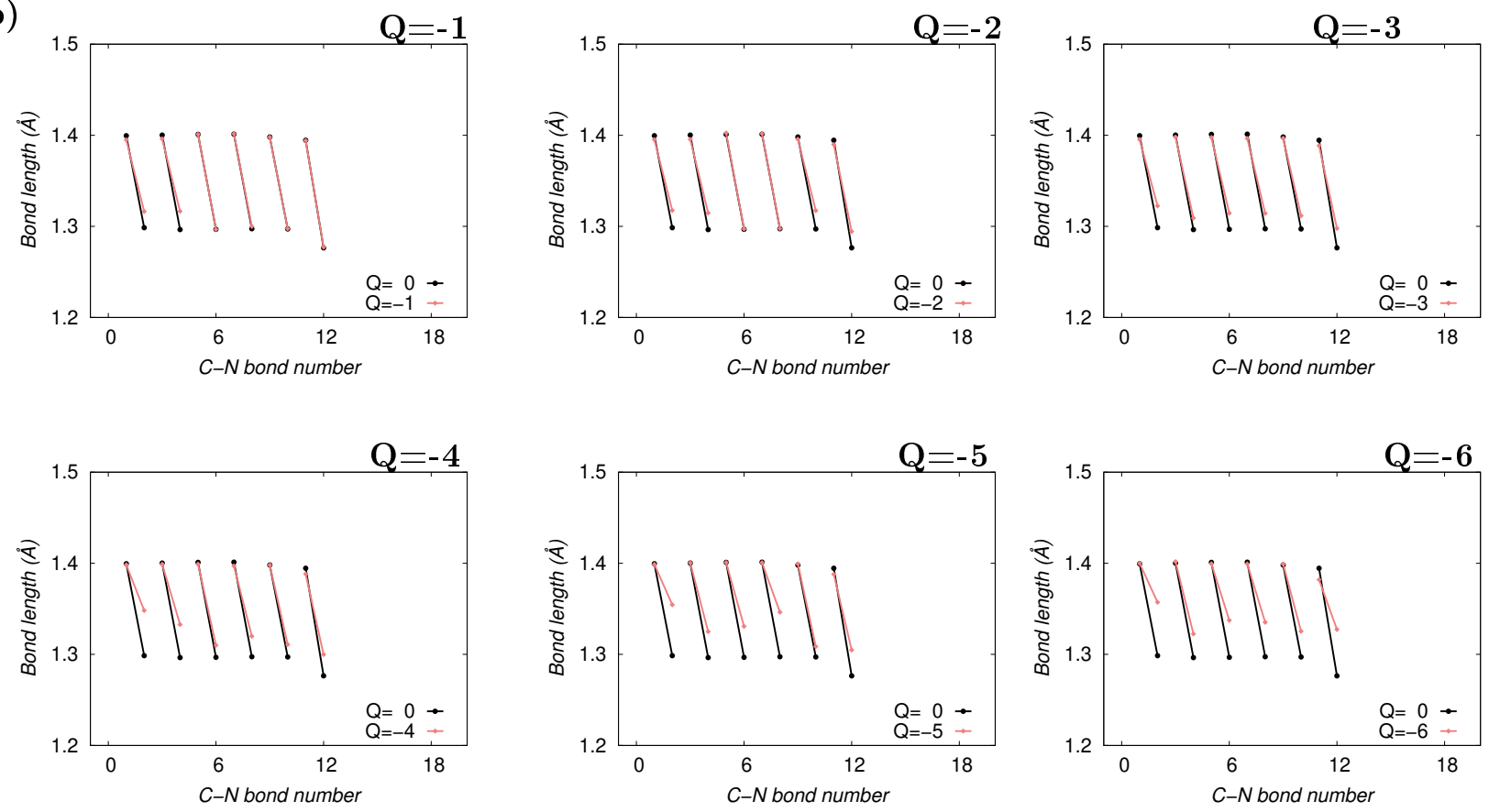

Figure S5: a) BBL chain where the imidazole ring are marked by red tikz and the $\mathrm{C}-\mathrm{N}$ bond number is also shown, b) Bond length alternation of $\mathrm{C}-\mathrm{N}$ bonds of the imidazole ring from $\mathrm{M}_{2}$ repeating unit of doped $\mathrm{BBL}(\mathrm{Q}=-1$ to -6$)$. 

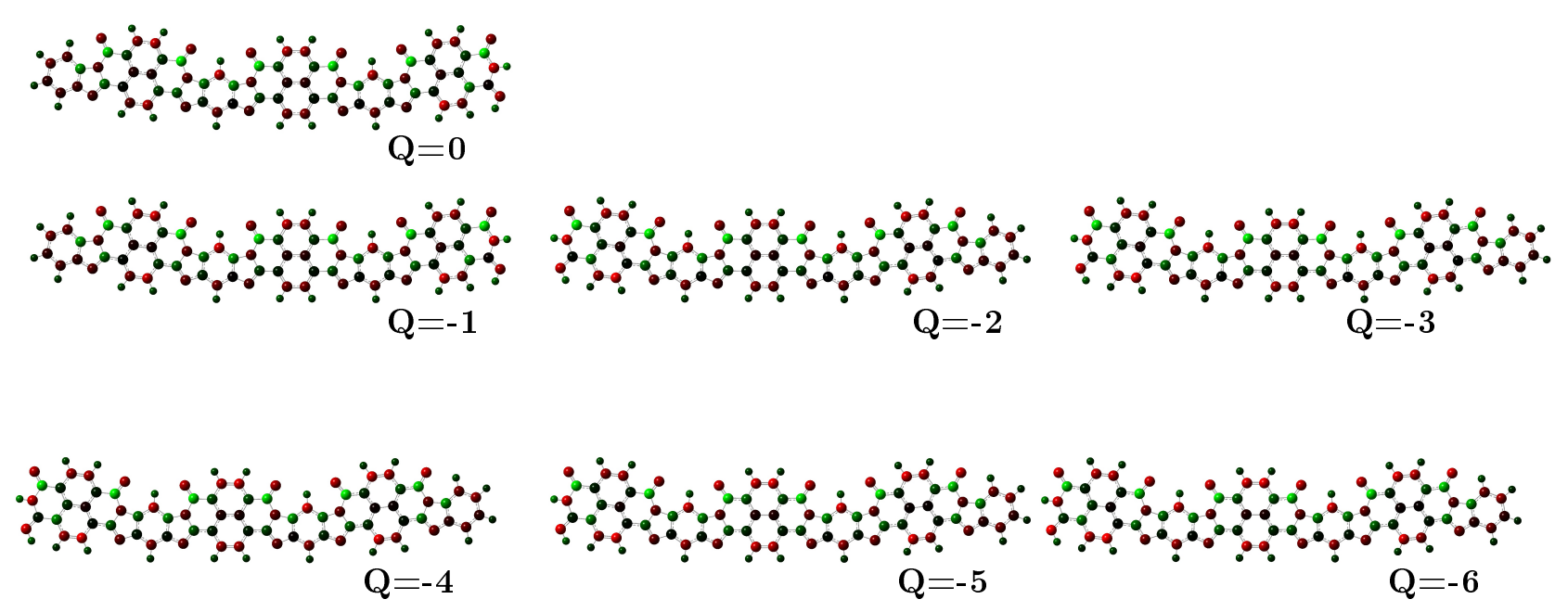

Figure S6: Charge distribution of undoped and doped BBL.

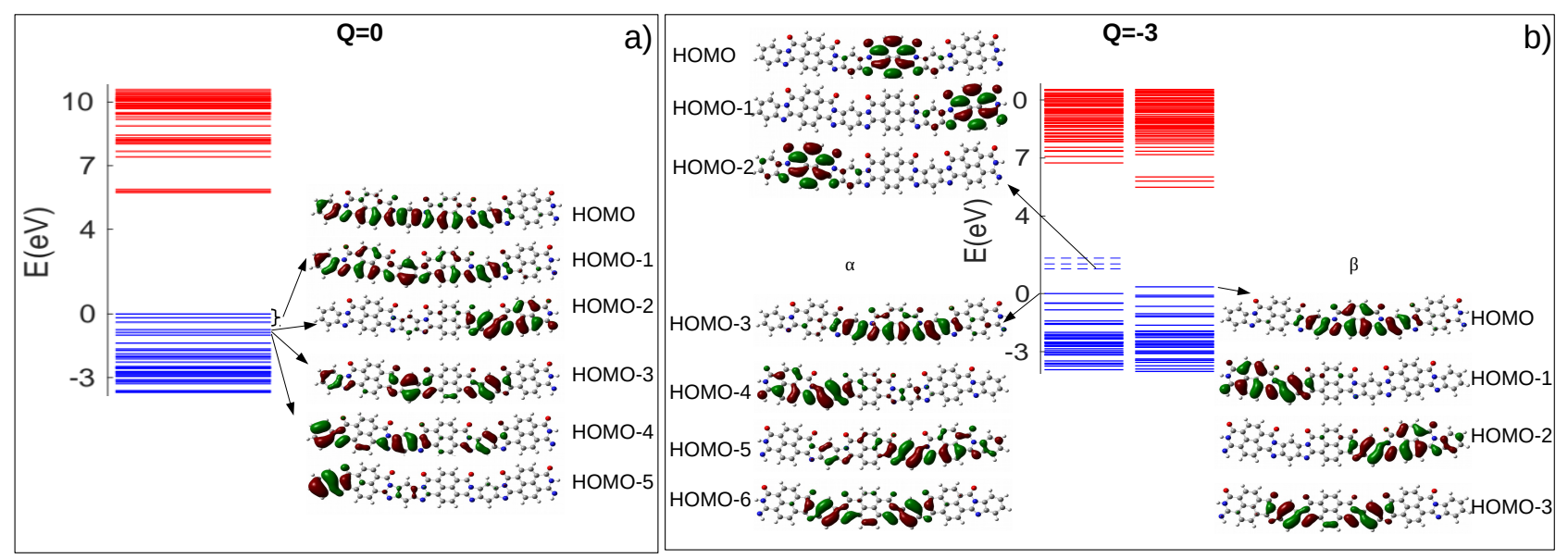

Figure S7: Band diagrams of a) undoped $(\mathrm{Q}=0)$ and $\mathrm{b})$ doped $\mathrm{BBL}(\mathrm{Q}=-3)$ are plotted and their occupied electronic states in the valence band are visualized. 


\section{Result of PEDOT}

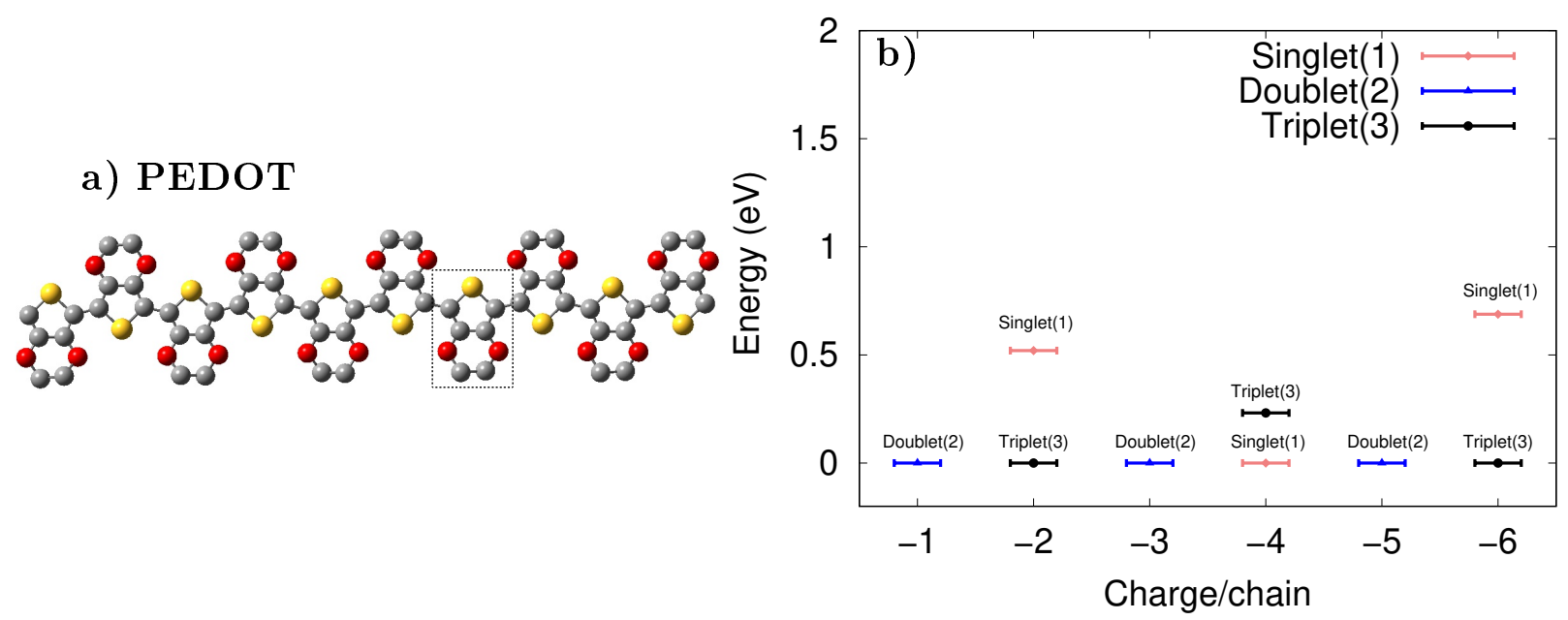

Figure S8: a) Schematic representation PEDOT polymer chain where carbon, oxygen, sulfur, nitrogen atoms are represented as grey, red, yellow and blue sphere, repectively. Hydrogen atoms are not shown for clarity. One monomer unit of PEDOT is marked by dotted line. b) Total energy of a PEDOT chain of different spin states for different charged states from -1 to -6 . 


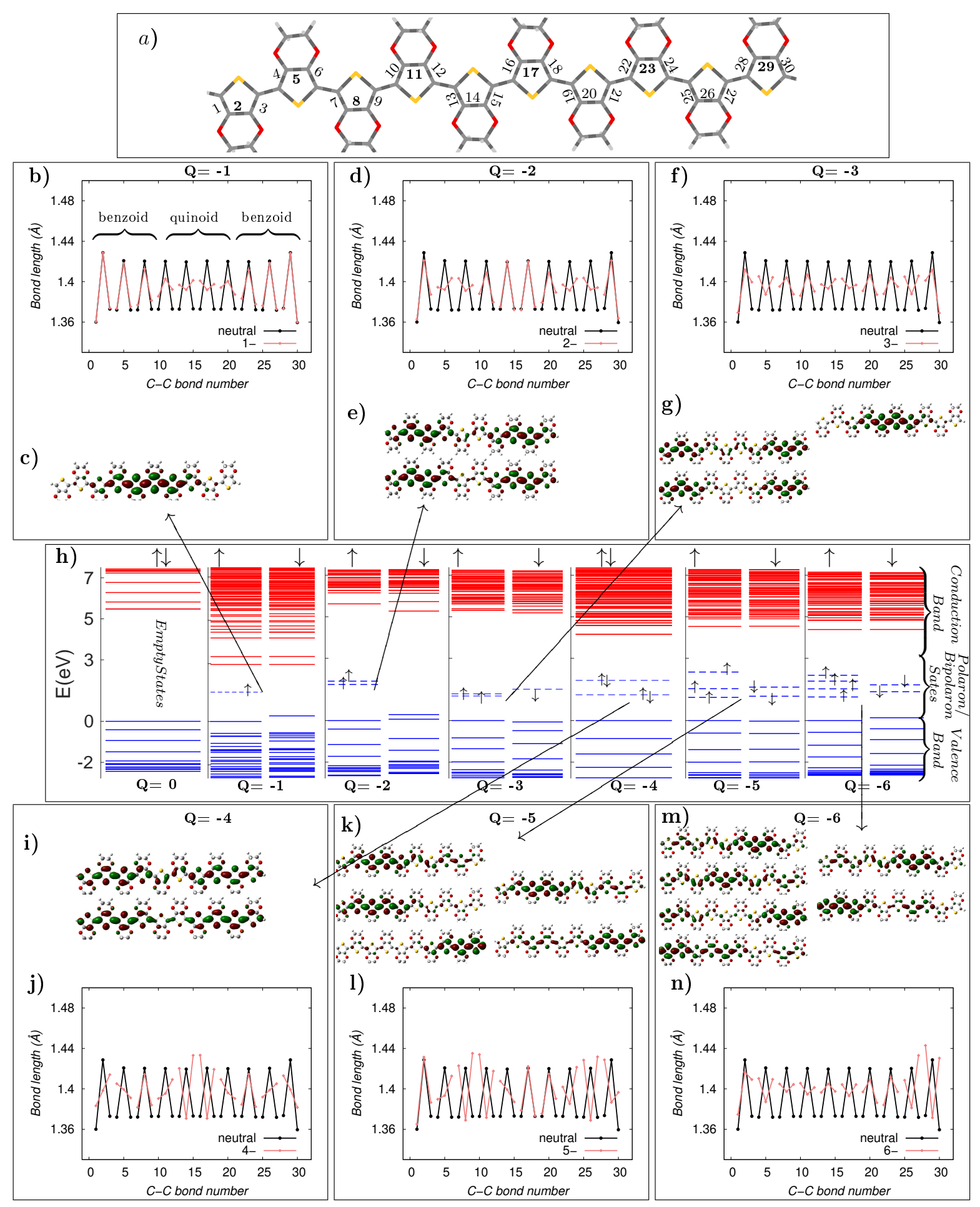

Figure S9: a) PEDOT chain: all the $\mathrm{C}-\mathrm{C}$ bond number which are used for calculation of bond length alternation are marked, b) d) f) i) k) m) Bond length alternation of the 6membered ring of doped PEDOT. c) e) g) j) l) n) The corresponding electron densities of polaron/bipolaron states are also shown for all the charged states $(\mathrm{Q}=-1,-2,-3,-4,-5,-6)$. h) Band diagrams of undoped $(\mathrm{Q}=0)$ and doped $\operatorname{PEDOT}(\mathrm{Q}=-1$ to -6$)$. Empty electronic states in the conduction band are plotted as red lines, occupied electronic states in the valence band are plotted as blue lines and the ocuupied polaron/bipolaron states are marked as dotted lines. Spin up and spin down electron levels are shown as uparrow and downarrow, respectively. 
a)

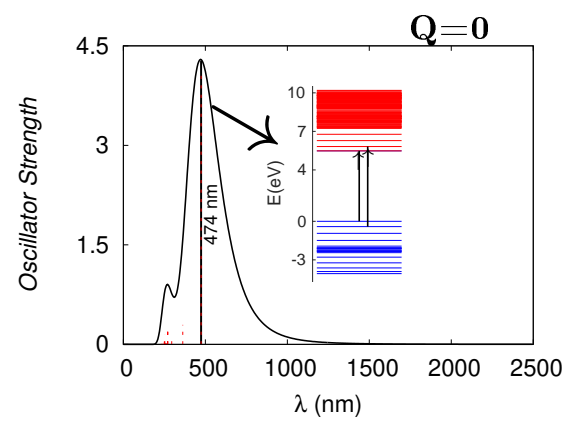

b)

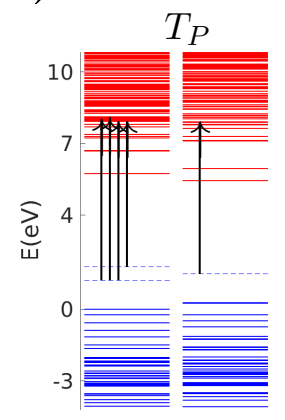

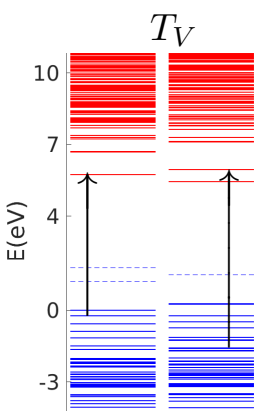

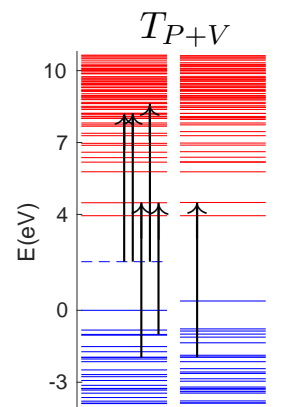

c)
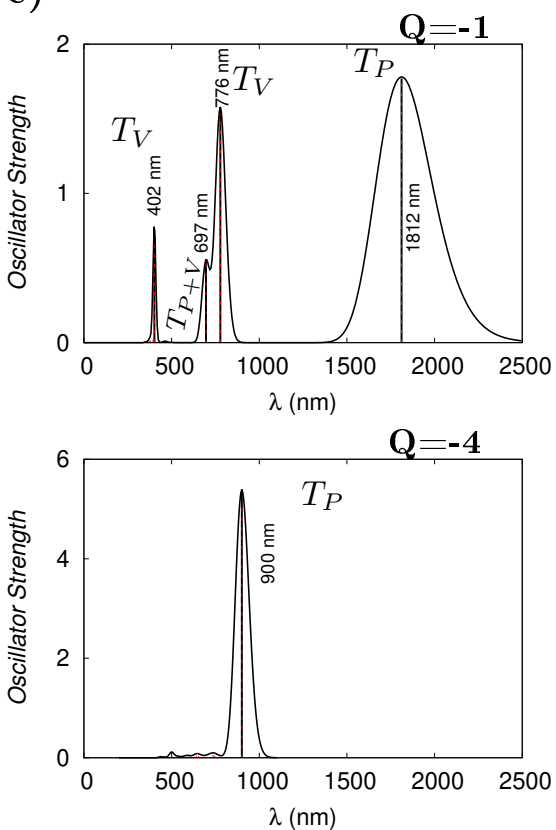
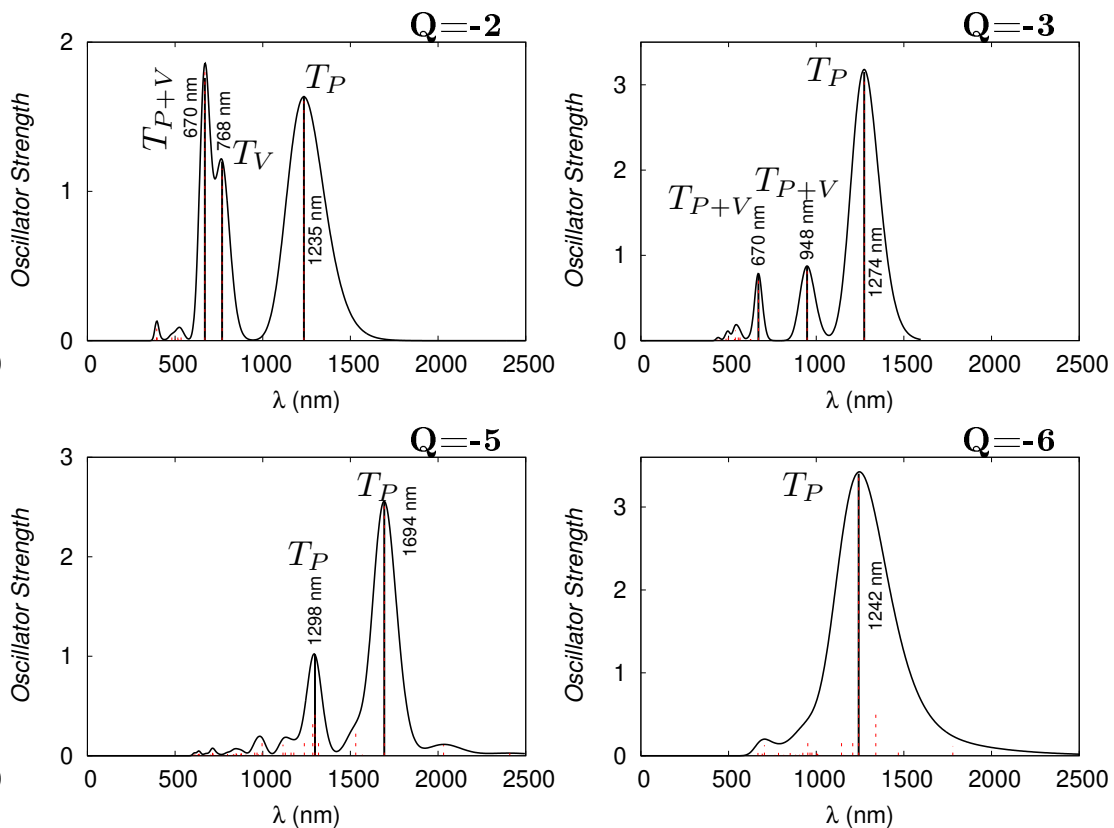

Figure S10: a) UV vis/NIR absorption spectra of a neutral PEDOT chain where the transition from valence band to conduction band corresponding to the absorbance peaks are also shown in the band structure, b) The absorption peaks due to the transition from polaronic/bipolaronic states to conduction band are named as $T_{P}$, transition from valence band to conduction band are named as $T_{V}$, from both the polaronic/bipolarnic and the other states of valence band to the conduction band are named as $\left.T_{P+V}, \mathrm{c}\right) \mathrm{UV}$ vis/NIR absorption spectra of a PEDOT chain for several doped states from $\mathrm{Q}=-1$ to -6 . 